\title{
Cataract Surgery in Patients with a Previous History of KAMRA Inlay Implantation: A Case Series
}

\author{
Majid Moshirfar - Tyler S. Quist • David F. Skanchy · Steven H. Linn • \\ Jordan Desautels · Phillip C. Hoopes Jr.
}

Received: March 10, 2017 / Published online: April 25, 2017

(C) The Author(s) 2017. This article is an open access publication

\section{ABSTRACT}

Introduction: The visual outcome of cataract surgery in patients with a previous history of KAMRA inlay implantation is an emerging issue for which limited research exists. The purpose of this study is to describe postoperative visual outcomes in this patient population.

Methods: This is a case series with retrospective chart review. Seven patients underwent cataract

Enhanced Content To view enhanced content for this article go to http://www.medengine.com/Redeem/ 3818F06066AEAC90.

M. Moshirfar $(\bowtie) \cdot$ S. H. Linn · P. C. Hoopes Jr. HDR Research Center, Hoopes Vision, Draper, UT, USA

e-mail: cornea2020@me.com

\section{Moshirfar}

Department of Ophthalmology and Visual Sciences, John A. Moran Eye Center, University of Utah

School of Medicine, Salt Lake City, UT, USA

T. S. Quist

University of Utah School of Medicine, Salt Lake

City, UT, USA

D. F. Skanchy

McGovern Medical School, The University of Texas

Health Science Center at Houston, Houston, TX, USA

J. Desautels

Tufts University School of Medicine, Boston, MA, USA surgery following KAMRA corneal inlay implantation. Visual acuity was assessed before and after cataract surgery. Keratometry was measured before and after KAMRA inlay implantation, and optical biometry was performed prior to cataract surgery.

Results: Postoperatively, uncorrected distance visual acuity (UDVA) was 20/20 in five (71\%) patients and $20 / 40$ or better in seven (100\%) patients, corrected distance visual acuity (CDVA) was $20 / 20$ or better in six (86\%) patients and 20/25 in seven (100\%) patients, and uncorrected near visual acuity (UNVA) was $\mathrm{J} 1$ in four $(57 \%)$ patients, $\mathrm{J} 2$ or better in six (86\%) patients, and J3 or better in seven (100\%) patients. There was no significant change in keratometry after KAMRA inlay implantation. Biometry calculations accurately predicted intraocular lens (IOL) power after cataract surgery. Surgeons did not report a significant change in the surgical technique of cataract surgery.

Conclusion: Patients who undergo cataract surgery with a previous history of KAMRA inlay implantation have good visual outcomes. Furthermore, intraocular lens (IOL) calculations accurately predict power, and there appears to be no significant change in surgical technique.

Keywords: Corneal inlay; KAMRA; Presbyopia; Small aperture inlay 


\section{INTRODUCTION}

Presbyopia is an age-related condition in which the lens of the eye loses elasticity, resulting in inability to focus on near objects. Today, vision impairment due to uncorrected presbyopia is a major cause of disability $[1,2]$. As life expectancy continues to rise in the general population, the prevalence of presbyopia will also likely increase.

The current surgical management of presbyopia generally includes lens procedures in eyes with cataracts and corneal procedures in eyes without cataracts, though other options such as refractive lens extraction may be considered depending on individual ocular characteristics. The latter involves corneal inlays, which are petite optical devices placed within the corneal stroma that function to improve near vision. The small aperture inlay (KAMRA, Acufocus Inc., Irvine, CA, USA) is a type of corneal inlay that has been shown to be effective in the treatment of presbyopia [3-7]. The device is an opaque disk with an outer diameter of $3.8 \mathrm{~mm}$, an inner diameter of $1.6 \mathrm{~mm}$, and a thickness of 5 microns. The aperture takes advantage of pinhole optics by reducing the opening of the eye to increase the depth of focus, thus improving near vision $[8,9]$.

Given that presbyopia often occurs during the 5th decade in life, patients who undergo inlay implantation may later develop cataracts, which require surgery. Other than a few case reports, limited published literature exists regarding cataract surgery in patients with a KAMRA inlay [10]. In this work, we present the visual outcomes of seven patients previously treated with KAMRA inlay, who later underwent phacoemulsification and intraocular lens (IOL) implantation.

\section{METHODS}

Seven patients underwent cataract surgery with a previous history of KAMRA inlay implantation. All patients involved were provided written informed consent in accordance with the tenets of the Declaration of Helsinki to having their data used for research purposes. This study was approved by the Hoopes, Durrie, Rivera Internal Review Board. All procedures followed were in accordance with the ethical standards of the responsible committee on human experimentation (institutional and national) and with the Helsinki Declaration of 1964, as revised in 2013. Regarding KAMRA inlay implantation, a femtosecond laser $(150-\mathrm{kHz}$ IntraLase iFS; Abbott Medical Optics Inc) was used for flap creation. Postoperatively, patients were treated with topical ofloxacin $0.3 \%$ (Ocuflox, Allergan Inc, Irvine, CA) for 1 week four times daily, prednisolone acetate 1.0\% (Pred Forte, Allergan Inc., Irvine, CA) for 1 week four times daily, and fluorometholone $0.1 \%$ (FML, Allergan Inc, Irvine, CA) for 3 weeks at a tapering dose starting at four times daily. Regarding cataract surgery, patients underwent optical biometry measurements for intraocular lens (IOL) power calculation via the ZEISS IOLMaster ${ }^{\circledR}$ (Carl Zeiss Meditec, Jena, Germany). All patients underwent phacoemulsification and posterior chamber IOL implantation with the KAMRA inlay left in place.

\section{CASE PRESENTATIONS}

For all seven patients, the uncorrected distance visual acuity (UDVA), corrected distance visual acuity (CDVA), uncorrected near visual acuity (UNVA), and manifest refraction spherical equivalent (MRSE) before and after cataract surgery are shown in Table 1. Postoperatively, UDVA was $20 / 20$ in five $(71 \%)$ patients and $20 / 40$ or better in seven $(100 \%)$ patients, while CDVA was $20 / 20$ or better in six $(86 \%)$ patients and $20 / 25$ in seven (100\%) patients. Postoperatively, UNVA was J1 in four (57\%) patients, J2 or better in six $(86 \%)$ patients, and J3 or better in seven $(100 \%)$ patients. No patients had perioperative complications. Given the similarity between cases, detailed information is provided for three cases, and their overall results are shown in Table 2.

\section{Case 1}

Case one is a 58-year-old white female with no prior ocular surgeries and a history of 
Table 1 Monocular visual acuity before cataract surgery and at 3 months

\begin{tabular}{|c|c|c|c|}
\hline Case & Measurement & $\begin{array}{l}\text { Before cataract } \\
\text { surgery }\end{array}$ & $\begin{array}{l}\text { After cataract } \\
\text { surgery }\end{array}$ \\
\hline \multirow[t]{4}{*}{1} & UDVA & $20 / 70$ & $20 / 20$ \\
\hline & CDVA & $20 / 40$ & $20 / 20$ \\
\hline & UNVA & $\mathrm{J} 5$ & $\mathrm{~J} 2$ \\
\hline & MRSE & 0.75 & 0.125 \\
\hline \multirow[t]{4}{*}{2} & UDVA & $20 / 40$ & $20 / 20$ \\
\hline & CDVA & $20 / 30$ & $20 / 15$ \\
\hline & UNVA & $\mathrm{J} 2$ & $\mathrm{~J} 1$ \\
\hline & MRSE & -0.125 & -0.75 \\
\hline \multirow[t]{4}{*}{3} & UDVA & $20 / 150$ & $20 / 40$ \\
\hline & CDVA & $20 / 25$ & $20 / 20$ \\
\hline & UNVA & $\mathrm{J} 1$ & $\mathrm{~J} 3$ \\
\hline & MRSE & -2.125 & -0.25 \\
\hline \multirow[t]{4}{*}{4} & UDVA & $20 / 30$ & $20 / 30$ \\
\hline & CDVA & $20 / 25$ & $20 / 25$ \\
\hline & UNVA & $\mathrm{J} 7$ & $\mathrm{~J} 1+$ \\
\hline & MRSE & -1.75 & -0.25 \\
\hline \multirow[t]{4}{*}{5} & UDVA & $20 / 150$ & $20 / 20$ \\
\hline & CDVA & $20 / 50$ & $20 / 20$ \\
\hline & UNVA & $\mathrm{J} 7$ & $\mathrm{~J} 1+$ \\
\hline & MRSE & -1.75 & -0.25 \\
\hline \multirow[t]{4}{*}{6} & UDVA & $20 / 30$ & $20 / 20$ \\
\hline & CDVA & $20 / 30$ & $20 / 20$ \\
\hline & UNVA & $\mathrm{J} 8$ & $\mathrm{~J} 1+$ \\
\hline & MRSE & 0.25 & -0.25 \\
\hline \multirow[t]{4}{*}{7} & UDVA & $20 / 40$ & $20 / 20$ \\
\hline & CDVA & $20 / 25$ & $20 / 20$ \\
\hline & UNVA & $\mathrm{J} 10$ & $\mathrm{~J} 2$ \\
\hline & MRSE & 1.625 & 0.25 \\
\hline
\end{tabular}

UDVA uncorrected distance visual acuity, CDVA corrected distance visual acuity, $U N V A$ uncorrected near visual acuity, MRSE manifest refraction spherical equivalent
Table 2 Keratometry, biometry values, biometry method, biometry formula, target power, and power achieved after cataract surgery for three cases

\begin{tabular}{llll}
\hline Measurement & \multicolumn{2}{l}{ Case } & \\
\cline { 2 - 4 } & $\mathbf{1}$ & $\mathbf{2}$ & $\mathbf{3}$ \\
\hline$K_{\mathrm{m}}$ before KAMRA & 43.7 & 45.6 & 44.2 \\
$\quad(\mathrm{D})$ & & & \\
$K_{\mathrm{m}}$ after KAMRA & 43.6 & 45.7 & 44.5 \\
$\quad(\mathrm{D})$ & & & \\
$K_{\mathrm{m} \text { before cataract }}$ & 43.4 & 46.0 & 44.5 \\
$\quad$ surgery & & & \\
AL (mm) & 23.49 & 22.23 & 23.99 \\
Cyl (D) & -1.17 & -0.75 & -0.82 \\
IOL & 22.0 & 20.5 & 19.5 \\
& ZCB00 & ZCB00 & ZCB00 \\
Formula used & Holladay & SRK/T & SRK/T \\
& 1 & & \\
Target power & -0.55 & -0.85 & -0.46 \\
Achieved power & 0.125 & -0.75 & -0.25 \\
\hline
\end{tabular}

$K$ average keratometry, $A L$ axial length, $C y l$ cylinder, $I O L$ intraocular lens

IOL MASTER $^{\circledR}$ (Carl Zeiss Meditec, Jena, Germany)

presbyopia who presented with a non-dominant eye UDVA of 20/15, a CDVA of 20/15, UNVA of J8, and manifest refraction at distance of $+0.50-0.50 \times 014$ with a near add of +2.25 . At the time of surgery, a lamellar flap was created that was $9.5 \mathrm{~mm}$ in diameter and 210 microns in depth. The patient had a 3-month postoperative UDVA of 20/15, CDVA of 20/15, UNVA of $\mathrm{J} 2$, and manifest refraction at distance of $+0.0-0.50 \times 170$.

Thirty months after KAMRA inlay implantation, the patient developed a $1+$ anterior cortical cataract in the right eye. Sixty months after KAMRA inlay implantation, the patient had a UDVA of 20/70, CDVA of 20/40, UNVA of J5, and manifest refraction of $+1.00-0.50 \times 014$ (Table 1). At the time of cataract surgery, a 3-mm corneal incision was made at the 9 o'clock position, and an AMO model ZCBOO 22.0 diopter lens was then inserted into the 
capsular bag. The surgeon did not report any changes from his standard surgical technique, the procedure lasted $15 \mathrm{~min}$, and there were no surgical complications. At 3 months postoperatively, the patient had a UDVA of 20/20, CDVA of 20/20, UNVA of J2, and manifest refraction of $+0.25-0.25 \times 120$ (Table 1 ). The target power using the Holladay 1 formula was $-0.55 \mathrm{D}$ and was most accurate at predicting the achieved power of +0.125 , a difference of +0.67 (Table 2). However, SRK/T was also accurate and predicted a power of -0.57 , a difference of +0.69 .

\section{Case 2}

Case two is a 56-year-old white male with no prior ocular surgeries and a history of presbyopia who presented with non-dominant eye UDVA of 20/15, CDVA of 20/15, UNVA of J8, and manifest refraction of $+0.25-0.50 \times 097$ and near add of 2.25. At the time of surgery, a lamellar flap was created that was $9.0 \mathrm{~mm}$ in diameter and 210 microns in depth. The patient had a 3-month postoperative UDVA of $20 / 15$, CDVA of $20 / 15$, UNVA of J4, and manifest refraction at distance of $+0.50-0.50$ $\times 005$.

Fifty-eight months after KAMRA inlay implantation, the patient developed a $1+$ anterior cortical cataract in the left eye. Sixty-three months after KAMRA inlay implantation, the patient had a UDVA of 20/40, CDVA of 20/30, UNVA of J2, and manifest refraction at distance of $+0.50-1.25 \times 164$ (Table 1). At the time of cataract surgery, a 3-mm corneal incision was made at the 3 o'clock position, and an AMO model ZCBO0 20.5 diopter lens was then inserted into the capsular bag. The surgeon did not report any changes from his standard surgical technique, the procedure lasted $25 \mathrm{~min}$, and there were no surgical complications. Three months postoperatively, the patient had a UDVA of 20/20, CDVA of $20 / 15$, UNVA of J1, and manifest refraction of -0.75 (Table 1). The target power using the SRK/T was $-0.85 \mathrm{D}$ and was most accurate at predicting the achieved power of -0.38 , a difference of $0.10 \mathrm{D}$ (Table 2).

\section{Case 3}

Case three is a 56-year-old white male with a no prior ocular surgeries and a history of presbyopia who presented with non-dominant eye UDVA of $20 / 25$, CDVA of 20/15, UNVA of J8, and manifest refraction at distance of $+0.25-0.75 \times 146$ and near add of 2.25. At the time of surgery, a lamellar flap was created that was $9.5 \mathrm{~mm}$ in diameter and 210 microns in depth. The patient had a 3-month postoperative UDVA of 20/20, CDVA of 20/16, UNVA of J1, and manifest refraction at distance of $+0.25-0.75 \times 163$.

Fifteen months after KAMRA inlay implantation, the patient developed a nuclear sclerotic cataract in the right eye. At 26 months status post KAMRA inlay implantation, the patient had a UDVA of 20/150, CDVA of 20/25, UNVA of $\mathrm{J} 1$, and manifest refraction at distance of $-1.75-0.75 \times 001$ (Table 1). At the time of cataract surgery, a corneal incision was made at the 9 o'clock position, and an AMO model ZCB00 19.5 diopter lens was then inserted into the capsular bag. The surgeon did not report any changes from his standard surgical technique, the procedure lasted $20 \mathrm{~min}$, and there were no surgical complications. At 3 months postoperatively, the patient had a UDVA of 20/40, CDVA of 20/20, UNVA of J3, and manifest refraction at distance of $+0.25-1.00 \times 164$ (Table 1). The target power using the SRK/T formula was $-0.46 \mathrm{D}$ and was most accurate at predicting the achieved power of -0.25 , a difference of $0.21 \mathrm{D}$ (Table 2).

\section{DISCUSSION AND CONCLUSIONS}

Cataracts and presbyopia are both age-related conditions that cause a decrease in visual acuity. To date, the published literature is limited concerning patients who underwent cataract surgery with a previous history of KAMRA inlay implantation [10]. As the number of presbyopic patients treated with corneal inlay increases, the number of patients requiring cataract surgery in eyes previously treated with KAMRA inlay will likely increase. This scenario poses several potential concerns for physicians as outlined by Tien-en Tan et al., including poor 
visual outcomes, inaccuracy of IOL power calculations, and the need to alter the surgical technique secondary to poor visualization [10].

Poor visual outcomes are a consideration in patients who undergo cataract surgery with a previous history of KAMRA inlay implantation. In this study, visual outcomes were good in all patients after cataract surgery with improvement in both distance and near vision. Postoperatively, all patients had good UDVA, CDVA, and UNVA. Similarly, Tien-en Tan et al. also reported good distance and near visual outcomes after cataract surgery with a mean postoperative UDVA of 20/30, CDVA of 20/25, and UNVA of J2 [10].

Another possible complication is inaccuracy of the IOL power calculation, which may be affected by changes in keratometry secondary to the previously implanted inlay. In this work, the mean change in keratometry was 0.17 $\mathrm{D} \pm 0.11$ (SD) after KAMRA inlay implantation $(n=3)$. This suggests that a negligible change in keratometry occurs after inlay implantation. Furthermore, IOL power calculations were accurate at predicting IOL power after surgery. Three formulas were used to calculate the target IOL power retrospectively, including Haigis, Holladay 1, and SRK/T. In case one, both the Holladay 1 and SRK/T formulae were similar in their prediction of IOL power, and in cases two and three, the SRK/T formula was best at predicting IOL power. The mean target IOL power was $-0.62 \mathrm{D} \pm 0.20(\mathrm{SD})$, the mean achieved IOL power was $-0.29 \mathrm{D} \pm 0.44(\mathrm{SD})$, and the mean difference in target and achieved IOL power was $0.32 \mathrm{D} \pm 0.31$ (SD) $(n=3)$. Tien-en Tan et al. also found the calculation of IOL power to be accurate. The author reported that the SRK/T formula was the most accurate formula for predicting the achieved power in emmetropic presbyopic patients [10]. However, the SRK/T formula may not apply to all patients with a previous history of karma inlay implantation who later undergo cataract surgery. A direct comparison of IOL power calculations may be confounded by varying axial length even more than keratometry in the patients evaluated. Given the small patient population in this study, no definitive correlation can be made between axial length and the most accurate formula, and no prior research has addressed which formula is most appropriate with regards to the karma inlay. Thus, we emphasize the need to look at all formulas based on the individual patient.

Regarding the mean difference of $+0.3 \mathrm{D}$ between the target and achieved IOL power reported in this study, there may be a tendency toward hyperopic surprise in this patient population. There are many factors that can cause drift in postoperative refraction including effective lens position, changes in biometry, and corneal stromal remodeling changes or inflammation due to the KAMRA inlay itself. To date, however, there is no published literature addressing the possibility of hyperopic shift associated with the karma inlay. Given our small patient sample size, it is difficult to say whether there truly is a trend toward hyperopia. Regardless, our mean difference between target and achieved IOL power falls within the level of accuracy previously reported for cataract surgery in patients without a previous history of karma inlay implantation. Consistent with our outcomes, Shah et al. reported that $77.7 \%$ (153 of 197) of eyes undergoing routine cataract surgery had a refractive outcome within $0.5 \mathrm{D}$ of target refraction and $91.4 \%$ within $1.0 \mathrm{D}$ without reporting any tendency toward hyperopic shift [11].

Another potential concern is poor visualization while performing cataract surgery secondary to the KAMRA inlay and the need to alter one's surgical technique. In this work, the mean operating time was $20 \mathrm{~min}(n=3)$. Surgeons reported no change to their normal surgical technique other than the need of additional ocular rotations during capsulorhexis and phacoemulsification to improve visualization during surgery. There was a 10-min difference in operative times between case 1 (15 $\mathrm{min})$ and case 2 (25 min). Cataract surgery was performed by a different surgeon in these cases, which may account for the length of each operation. Otherwise, no additional information in the operative reports explains this difference. Similarly to our results, Tien-en Tan et al. reported a mean operating time of approximately $15 \mathrm{~min}$ and no significant changes to the regular surgical technique other than additional ocular rotations to improve 
visualization [10]. Thus, no significant change in surgical technique appears to be necessary.

In conclusion, cataract surgery is a viable option in patients with a previous history of KAMRA inlay. Our research suggests that visual outcomes after cataract surgery are good with an improvement in near and distance vision. Previous KAMRA inlay implantation results in a negligible change in keratometry and does not affect the accuracy of IOL power calculations. No significant change in surgical technique appears to be needed during uncomplicated cataract surgery. Given our small sample size and the limited amount of published literature regarding cataract surgery following KAMRA inlay implantation, larger studies with longer follow-up are necessary to confirm our observations.

\section{ACKNOWLEDGMENTS}

No funding or sponsorship was received for this study or publication of this article. All named authors meet the International Committee of Medical Journal Editors (ICMJE) criteria for authorship for this manuscript, take responsibility for the integrity of the work as a whole, and have given final approval to the version to be published.

Disclosures. Majid Moshirfar, Tyler S. Quist, David F. Skanchy, Steven H. Linn, Jordan Desautels, and Phillip C. Hoopes Jr have nothing to disclose.

Compliance with Ethics Guidelines. All patients involved were provided written informed consent in accordance with the tenets of the Declaration of Helsinki to having their data used for research purposes. This study was approved by the Hoopes, Durrie, Rivera Internal Review Board. All procedures followed were in accordance with the ethical standards of the responsible committee on human experimentation (institutional and national) and with the Helsinki Declaration of 1964, as revised in 2013.

Data Availability. All data generated or analyzed during this study are included in the tables provided in this published article.
Open Access. This article is distributed under the terms of the Creative Commons Attribution-NonCommercial 4.0 International License (http://creativecommons.org/licenses/ by-nc/4.0/), which permits any noncommercial use, distribution, and reproduction in any medium, provided you give appropriate credit to the original author(s) and the source, provide a link to the Creative Commons license, and indicate if changes were made.

\section{REFERENCES}

1. Frick KD, Joy SM, Wilson DA, Naidoo KS, Holden BA. The global burden of potential productivity loss from uncorrected presbyopia. Ophthalmol. 2015;122(8):1706-10.

2. Mcdonnell PJ, Lee P, Spritzer K, Lindblad AS, Hays RD. Associations of presbyopia with vision-targeted health-related quality of life. Arch Ophthalmol. 2003;121(11):1577-81.

3. Seyeddain O, Riha W, Hohensinn M, Nix G, Dexl AK, Grabner G. Refractive surgical correction of presbyopia with the AcuFocus small aperture corneal inlay: two-year follow-up. J Refract Surg. 2010;26(10):707-15.

4. Jalali S, der Aus W, Shaarawy T. AcuFocus corneal inlay to correct presbyopia using femto-LASIK. One year results of a prospective cohort study. Klin Monbl Augenheilkd. 2016;233(4):360-4.

5. Dexl AK, Jell G, Strohmaier C, Seyeddain O, Riha W, Rückl T, et al. Long-term outcomes after monocular corneal inlay implantation for the surgical compensation of presbyopia. J Cataract Refract Surg. 2015;41(3):566-75.

6. Seyeddain O, Bachernegg A, Riha W, Rückl T, Reitsamer H, Grabner G, et al. Femtosecond laser-assisted small-aperture corneal inlay implantation for corneal compensation of presbyopia: two-year follow-up. J Cataract Refract Surg. 2013;39(2):234-41.

7. Dexl AK, Seyeddain O, Riha W, Rückle T, Bachernegg A, Emesz $M$, et al. Reading performance and patient satisfaction after corneal inlay implantation for presbyopia correction: two-year follow-up. J Cataract Refract Surg. 2012;38(10):1808-16.

8. Papadopoulos PA, Papadopoulos AP. Current management of presbyopia. Middle East Afr J Ophthalmol. 2014;21(1):10-7. 
9. Dexl AK, Seyeddain O, Riha W, Hohensinn M, Rückl T, Reischl V, et al. One-year visual outcomes and patient satisfaction after surgical correction of presbyopia with an intracorneal inlay of a new design. J Cataract Refract Surg. 2012;38(2):262-9.

10. Tan TE, Mehta JS. Cataract surgery following KAMRA presbyopic implant. Clin Ophthalmol. 2013;7:1899-903.
11. Shah S, Peris-Martinez C, Reinhard T, Vinciguerra P. Visual outcomes after cataract surgery: multifocal versus monofocal intraocular lenses. J Refract Surg. 2015;31(10):658-66. 